\title{
AN UNEXPECTED PROPERTY OF ODD ORDER DERIVATIVES OF HARDY'S FUNCTION
}

\author{
Philippe Blanc
}

Communicated by Aleksandar Ivić

Abstract. Assuming the Riemann hypothesis, we show that the odd order derivatives of Hardy's function have, under some condition, an unexpected behavior for large values of $t$.

\section{Introduction and main result}

Let $\zeta$ be the Riemann zeta function, and $Z$ the Hardy function defined by

$$
Z(t)=e^{i \theta(t)} \zeta\left(\frac{1}{2}+i t\right)
$$

where

$$
\theta(t)=\arg \left(\pi^{-i \frac{t}{2}} \Gamma\left(\frac{1}{4}+i \frac{t}{2}\right)\right)
$$

and the argument is defined by continuous variation of $t$ starting with the value 0 at $t=0$. It can be shown [6] that

$$
\theta(t)=\frac{t}{2} \log \frac{t}{2 \pi}-\frac{t}{2}-\frac{\pi}{8}+O\left(\frac{1}{t}\right)
$$

The real zeros of $Z$ coincide with the zeros of $\zeta$ located on the line of real part $\frac{1}{2}$. If the Riemann hypothesis is true, then the number of zeros of $Z$ in the interval ] $0, t]$ is given by $[6]$

$$
N(t)=\frac{1}{\pi} \theta(t)+1+S(t)
$$

where $S(t)=\frac{1}{\pi} \arg \zeta\left(\frac{1}{2}+i t\right)$ if $t$ is not a zero of $Z$ and $\arg \zeta\left(\frac{1}{2}+i t\right)$ is defined by continuous variation along the straight lines joining $2,2+i t$ and $\frac{1}{2}+i t$ starting

2010 Mathematics Subject Classification: 11M26.

Key words and phrases: Riemann zeta function, distribution of zeros, Hardy's function.

Dedicated to Professor Jean Descloux on the occasion of his 80th birthday. 
with the initial value $\arg \zeta(2)=0$. If $t$ is a zero of $Z$ we set $S(t)=\lim _{\epsilon \rightarrow 0_{+}} S(t+\epsilon)$. Let us choose $T$, say $T=500$, and let us plot the graphs of functions

$$
f_{2 k-1}(t):=(-1)^{k+1} \frac{Z^{(2 k-1)}(t)}{\theta^{\prime}(T)^{2 k-1}}
$$

for $k=1, \ldots, 5$ on the interval $[T-10, T+10]$. Observe that the term $1 / \theta^{\prime}(T)^{2 k-1}$ is just a scaling factor. These graphs show that the functions $(-1)^{k+1} Z^{(2 k-1)}$ have

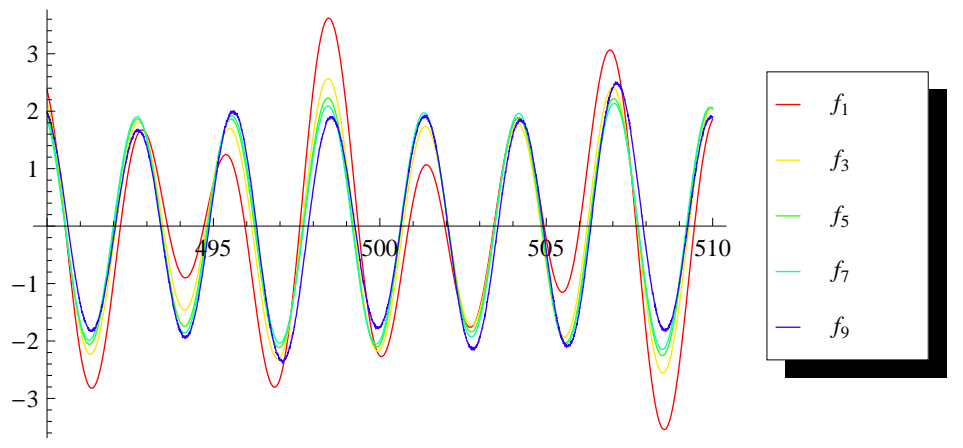

FiguRE 1. Graphs of $f_{2 k-1}$ for $k=1, \ldots, 5$

generally the same signs, at least for small values of $k$. This can be explained heuristically by a formula due to Lavrik [8], which asserts that for $t$ sufficiently large and $1 \leqslant k \leqslant \frac{1}{4} \log t$, we have, uniformly in $k$,

$$
\begin{aligned}
Z^{(2 k-1)}(t)=2(-1)^{k} \sum_{1 \leqslant n \leqslant \sqrt{t / 2 \pi}} \frac{1}{\sqrt{n}}\left(\theta^{\prime}(t)-\log n\right)^{2 k-1} \sin (\theta(t)-t \log n) & +O\left(t^{-\frac{1}{4}}\left(\frac{3}{2} \log t\right)^{2 k}\right) .
\end{aligned}
$$

Let us denote by $M=M(T)$ the largest integer $r$, possibly infinite, such that

$$
\operatorname{sign}\left((-1)^{k+1} Z^{(2 k-1)}(T)\right)=\operatorname{sign}\left(Z^{\prime}(T)\right) \quad \text { for } k=1,2, \ldots, r .
$$

For some $T$ the values of $M$ are surprisingly large. Using Mathematica we compute $Z$ with high precision and get for example $M(100)=26, M(1000.4)=138$ and $M(9999.5)=402$.

Now let $T$ be large enough such that $Z(T)>0$ and let $\gamma_{k}$, where $k \neq 0$, be the zeros of $Z$ ordered in increasing order, taking their multiplicities into account, and numbered so that $\cdots \leqslant \gamma_{-2} \leqslant \gamma_{-1}<T<\gamma_{1} \leqslant \gamma_{2} \leqslant \cdots$. Further, let $4(\log \log T)^{-1} \leqslant a \leqslant \sqrt{T}$ such that $T+a$ and $T-a$ are not zeros of $Z$ and, finally, let $m, n \geqslant 1$ such that $\gamma_{-m-1}<T-a<\gamma_{-m}$ and $\gamma_{n}<T+a<\gamma_{n+1}$. Note that the existence of $m, n \geqslant 1$ is an immediate consequence of a result of Goldston and Gonek [4]. We assume that $(-1)^{m} Z^{\prime}(T-a) \geqslant 0$ and $(-1)^{n} Z^{\prime}(T+a) \leqslant 0$ and we 
denote by $K=K(T, a)$ the largest integer $r$, possibly infinite, such that

$$
(-1)^{m+k+1} Z^{(2 k-1)}(T-a) \geqslant 0 \text { and }(-1)^{n+k+1} Z^{(2 k-1)}(T+a) \leqslant 0
$$

for $k=1,2, \ldots, r$. For some $T$ and $a$, the values of $K$ are also surprisingly large: $K(109.3,9.4)=21, K(1070.1,8.5)=108$ and $K(10025.5,9.8)=408$. The goal of this paper is to give a conditional upper bound for $K$.

It should be observed that if we replace $Z(t)$ by $\cos t$ and choose $T=0$ and $a=l \pi+\frac{\pi}{4}$ where $l \in \mathbb{N}^{*}$, then $m=n=l$ and conditions (1.2) hold for every $k$ and hence $K=\infty$.

We now define a quantity which appears in our main result. By Lavrik's formula [8], for $t$ sufficiently large and $0 \leqslant k \leqslant \frac{1}{4} \log t$, we have, uniformly in $k$,

$$
\begin{aligned}
Z^{(2 k)}(t)=2(-1)^{k} \sum_{1 \leqslant n \leqslant \sqrt{t / 2 \pi}} \frac{1}{\sqrt{n}}\left(\theta^{\prime}(t)-\log n\right)^{2 k} \cos (\theta(t) & -t \log n) \\
& +O\left(t^{-\frac{1}{4}}\left(\frac{3}{2} \log t\right)^{2 k+1}\right)
\end{aligned}
$$

and using $\theta^{\prime}(t)=\frac{1}{2} \log \frac{t}{2 \pi}+O\left(1 / t^{2}\right)$ we get

$$
\begin{aligned}
&\left|Z^{(2 k)}(t)\right|=\theta^{\prime}(t)^{2 k}\left(2 \sum_{1 \leqslant n \leqslant \sqrt{t / 2 \pi}} \frac{1}{\sqrt{n}}\left(1-\frac{\log n}{\theta^{\prime}(t)}\right)^{2 k} \cos (\theta(t)-t \log n)\right) \\
&+O\left(3^{2 k} \theta^{\prime}(t)^{2 k} t^{-\frac{1}{4}} \log t\right) .
\end{aligned}
$$

As Ivić says in [5], it is difficult to get good uniform bounds for $Z^{(2 k)}(t)$ from (1.3). Nevertheless, when $Z(T)$ is large, relation (1.3) suggests that

$$
\int_{T-a}^{T+a}\left(Z^{(2 k)}(t)\right)^{2} d t=2 a\left(c_{2 k} \theta^{\prime}(T)^{2 k} Z(T)\right)^{2}
$$

where $c_{2 k}$ is small. For the aforementioned computations, we used the results of Kotnik [7] and chose $T$ in a neighborhood of $10^{2}, 10^{3}$ and $10^{4}$ and $a$ approximately equal to 10 such that $Z(T)$ and $K(T, a)$ are large. We obtain $c_{42}=0.56 \ldots$ and $c_{216}=0.34 \ldots$ which show that $c_{2 k}$ can be small even for some $k>\frac{1}{4} \log T$ despite the fact that, for fixed $T$, the sequence $c_{2 k}$ is unbounded. Numerical experiments indicate that Lavrik's formula is probably true for larger values of $k$ with a better error term.

TheOrem 1.1. For $T$ large enough, let $4(\log \log T)^{-1} \leqslant a \leqslant \sqrt{T}, K$ be the number defined in the introduction, $\Delta S=S(T+a)-S(T-a)$ and further let $K^{*}=\frac{a}{\pi} \log T+\Delta S$. If the Riemann hypothesis is true and if $\Delta S \geqslant 1$, then

$$
K \leqslant \max \left(\frac{a}{2 \pi} A_{K, T} \frac{\log T}{\Delta S}\left(1+O\left(\frac{\log \log \log T}{\log \log T}\right)\right), K^{*} \log K^{*}\right)
$$

where

$$
A_{K, T}=\log c_{2 K}+\Delta S \log 2+\frac{1}{\sqrt{2}} \frac{\log T}{\log \log T}
$$


For $T$ large enough such that $Z(T)$ is large in the sense of [7], numerical experiments show that the bound (1.5) is probably true without the term $\log c_{2 K}$. If this is the case and if we neglect the big $\mathrm{O}$ in (1.5) and choose $T \leqslant 10^{50}$ and $a \leqslant 1$ such that $\Delta S=1$, we get $K \leqslant 327$. Note that for the three values of $K(T, a)$ already given, we have $\Delta S<0$. This suggests that the behaviour of $K$ is different according to $\Delta S \leqslant 0$ or $\Delta S \geqslant 1$. This is unexpected.

This work stems from an observation of Ivić [5] about the values of the derivatives of $Z$ in a neighborood of points where $|Z|$ attains a large value. Some of the material used in our proof has been published by the author in [1].

The notations used in this paper are standard: $\lfloor x\rfloor$ stand for the usual floor function and $\{x\}:=x-\lfloor x\rfloor$. Bernoulli and Chebyshev polynomials of degree $n$ are denoted by $B_{n}(x)$ and $T_{n}(x)$; they are defined by

$$
\int_{x}^{x+1} B_{n}(t) d t=x^{n} \text { and } T_{n}(\cos \theta)=\cos n \theta .
$$

The organization of this paper is as follows: In Section 2 we prove the key identity, a property of the derivatives of Bernoulli polynomials and preparatory lemmas. Section 3 is devoted to the proof of our main result.

\section{Preliminary results}

We first prove an identity which will be used later to establish a relation between the value of a function $f \in C^{2 r}[-a, a]$ at 0 , the zeros of $f$ and the values of its derivatives of odd order on the boundaries of the interval.

LEMma 2.1. Let $-a<x_{-m}<\cdots<x_{-1}<x_{0}<x_{1}<\cdots<x_{n}<a$ and for $l=1,2, \ldots$ let $\Psi_{2 l-1}$ be the function defined on $[-a, a]$ by

$$
\Psi_{2 l-1}(x)=\frac{(4 a)^{2 l-1}}{(2 l) !} \sum_{k=-m}^{n} \mu_{k}\left(B_{2 l}\left(\frac{1}{2}+\frac{x+x_{k}}{4 a}\right)+B_{2 l}\left(\left\{\frac{x-x_{k}}{4 a}\right\}\right)\right)
$$

where $\sum_{k=-m}^{n} \mu_{k}=0$. Then for $f \in C^{2 r}[-a, a]$ where $r \geqslant 1$, we have the identity

$$
\begin{aligned}
\sum_{k=-m}^{n} \mu_{k} f\left(x_{k}\right)=\sum_{k=1}^{r} f^{(2 k-1)}(a) \Psi_{2 k-1}(a) & -\sum_{k=1}^{r} f^{(2 k-1)}(-a) \Psi_{2 k-1}(-a) \\
& -\int_{-a}^{a} f^{(2 r)}(x) \Psi_{2 r-1}(x) d x
\end{aligned}
$$

ProOF. By definition the function $\Psi_{2 r-1}$ is $C^{2 r-2}$, piecewise polynomial and the relation $B_{l}^{\prime}(x)=l B_{l-1}(x)$ for $l=1,2, \ldots$ leads to

$$
\Psi_{2 r-1}^{(j)}(x)=\frac{(4 a)^{2 r-j-1}}{(2 r-j) !} \sum_{k=-m}^{n} \mu_{k}\left(B_{2 r-j}\left(\frac{1}{2}+\frac{x+x_{k}}{4 a}\right)+B_{2 r-j}\left(\left\{\frac{x-x_{k}}{4 a}\right\}\right)\right)
$$

for $j=1, \ldots, 2 r-1$ and $x \neq x_{-m}, \ldots, x_{n}$ if $j=2 r-1$. This implies that

$$
\Psi_{2 r-1}^{(2 r-2 j)}=\Psi_{2 j-1} \quad \text { for } j=1,2, \ldots, r-1
$$

and that 


$$
\begin{aligned}
& \Psi_{2 r-1}^{(2 r-2 j+1)}( \pm a) \\
& =\frac{(4 a)^{2 j-2}}{(2 j-1) !} \sum_{k=-m}^{n} \mu_{k}\left(B_{2 j-1}\left(\frac{1}{2}+\frac{ \pm a+x_{k}}{4 a}\right)+B_{2 j-1}\left(\left\{\frac{ \pm a-x_{k}}{4 a}\right\}\right)\right) \\
& =\frac{(4 a)^{2 j-2}}{(2 j-1) !} \sum_{k=-m}^{n} \mu_{k}\left(B_{2 j-1}\left(\frac{1}{2}+\frac{ \pm a+x_{k}}{4 a}\right)+B_{2 j-1}\left(\frac{1}{2}-\frac{ \pm a+x_{k}}{4 a}\right)\right) \\
& =0 \text { for } j=1,2, \ldots, r .
\end{aligned}
$$

Further for $x \neq x_{-m}, \ldots, x_{n}$ we have

$$
\begin{aligned}
\Psi_{2 r-1}^{(2 r-1)}(x) & =\sum_{k=-m}^{n} \mu_{k}\left(B_{1}\left(\frac{1}{2}+\frac{x+x_{k}}{4 a}\right)+B_{1}\left(\left\{\frac{x-x_{k}}{4 a}\right\}\right)\right) \\
& =\sum_{k=-m}^{n} \mu_{k}\left(\frac{x+x_{k}}{4 a}+\left\{\frac{x-x_{k}}{4 a}\right\}-\frac{1}{2}\right)
\end{aligned}
$$

and as $\sum_{k=-m}^{n} \mu_{k}=0$ the function $\Psi_{2 r-1}^{(2 r-1)}$ is piecewise constant. Explicitly, for $x \in] x_{j}, x_{j+1}[$, we get

$\Psi_{2 r-1}^{(2 r-1)}(x)=\sum_{k=-m}^{j} \mu_{k}\left(\frac{x}{2 a}-\frac{1}{2}\right)+\sum_{k=j+1}^{n} \mu_{k}\left(\frac{x}{2 a}+1-\frac{1}{2}\right)=\sum_{k=j+1}^{n} \mu_{k}=-\sum_{k=-m}^{j} \mu_{k}$

which leads to

$\int_{x_{j}}^{x_{j+1}} f^{\prime}(x) \Psi_{2 r-1}^{(2 r-1)}(x) d x=-\left(\sum_{k=-m}^{j} \mu_{k}\right)\left(f\left(x_{j+1}\right)-f\left(x_{j}\right)\right)$ for $j=-m, \ldots, n-1$.

Summing these equalities and using that $\Psi_{2 r-1}^{(2 r-1)}=0$ on the intervals $\left[-a, x_{-m}\right.$ [ and $\left.] x_{n}, a\right]$, which follows from (2.4), we have

$$
\sum_{k=-m}^{n} \mu_{k} f\left(x_{k}\right)=\int_{-a}^{a} f^{\prime}(x) \Psi_{2 r-1}^{(2 r-1)}(x) d x
$$

and we complete the proof by integrating $2 r-1$ times the right-hand side by parts taking into account relations (2.2) and (2.3).

For further use we recall some elementary facts concerning the divided differences.

Lemma 2.2. Let $I=]-T, T\left[, f \in C^{m+n}(I)\right.$ and let $g$ be the function defined for pairwise distinct numbers $t_{-m}, \ldots, t_{n} \in I$ by

$$
g\left(t_{-m}, \ldots, t_{n}\right)=\sum_{k=-m}^{n} \frac{f\left(t_{k}\right)}{\prod_{\substack{m \leqslant j \leqslant n \\ j \neq k}}\left(t_{k}-t_{j}\right)} .
$$

Then 
a) The function $g$ has a continuous extension $g^{*}$ defined for $t_{-m}, \ldots, t_{n} \in I$ and there exists $\eta=\eta\left(t_{-m}, \ldots, t_{n}\right) \in I$ such that

$$
g^{*}\left(t_{-m}, \ldots, t_{n}\right)=\frac{f^{(m+n)}(\eta)}{(m+n) !} .
$$

b) Let $y_{0}, y_{1}, \ldots, y_{l}$ be the distinct values of $t_{-m}, \ldots, t_{n}$ considered as fixed and let $r_{k}$ be the number of index $j$ such that $t_{j}=y_{k}$. Then there exist $\alpha_{k, i}$ depending on $y_{0}, y_{1}, \ldots, y_{l}$ such that

$$
g^{*}\left(t_{-m}, \ldots, t_{n}\right)=\sum_{k=0}^{l} \sum_{i=0}^{r_{k}-1} \alpha_{k, i} f^{(i)}\left(y_{k}\right) .
$$

Proof. Assertion a) is a consequence of the representation formula

$$
\begin{aligned}
& \text { 2.5) } \quad g\left(t_{-m}, \ldots, t_{n}\right)= \\
& \int_{0}^{1} d \tau_{1} \int_{0}^{\tau_{1}} d \tau_{2} \cdots \int_{0}^{\tau_{m+n-1}} f^{(m+n)}\left(t_{-m}+\sum_{k=1}^{m+n} \tau_{k}\left(t_{-m+k}-t_{-m+k-1}\right)\right) d \tau_{m+n} .
\end{aligned}
$$

A proof of $b$ ) is given in [9].

In the next lemma we indicate the choice of coefficients $\mu_{k}$ for which the identity of Lemma 2.1 is of practical use for large values of $a$. The main reason of this choice will appear in the proof of (2.11) in Lemma 2.9.

LEMMA 2.3. Let $\Psi_{2 l-1}$ be defined for pairwise distinct $\left.x_{-m}, \ldots, x_{n} \in\right]-a, a[$ by

$$
\Psi_{2 l-1}\left(x_{-m}, \ldots, x_{n}, x\right)=\frac{(4 a)^{2 l-1}}{(2 l) !} \sum_{k=-m}^{n} \mu_{k}\left(B_{2 l}\left(\frac{1}{2}+\frac{x+x_{k}}{4 a}\right)+B_{2 l}\left(\left\{\frac{x-x_{k}}{4 a}\right\}\right)\right)
$$

where

$$
\mu_{k}=\frac{\alpha_{k}}{\alpha_{0}} \text { and } \frac{1}{\alpha_{k}}=\prod_{\substack{m \leqslant j \leqslant n \\ j \neq k}}\left(\sin \left(\pi \frac{x_{k}}{2 a}\right)-\sin \left(\pi \frac{x_{j}}{2 a}\right)\right) \quad \text { for } k=-m, \ldots, n .
$$

Then

a) For $l \geqslant 1$ the functions $\Psi_{2 l-1}(\cdot, \ldots, \cdot, \pm a)$ have continuous extensions $\Psi_{2 l-1}^{*}(\cdot, \ldots, \cdot, \pm a)$ defined for $\left.x_{-m}, \ldots, x_{n} \in\right]-a, a[$.

b) If $2 l \geqslant m+n+2$ the function $\Psi_{2 l-1}$ has a continuous extension $\Psi_{2 l-1}^{*}$ defined for $\left.x_{-m}, \ldots, x_{n} \in\right]-a, a[$ and $x \in[-a, a]$.

c) If $2 r \geqslant m+n+2$ and $f \in C^{2 r}[-a, a]$ is defined on $[-a, a]$ and vanishes at $x_{k}$ with $k \neq 0$ where $-a<x_{-m} \leqslant \ldots \leqslant x_{-1}<x_{0}<x_{1} \leqslant \ldots \leqslant x_{n}<a$ and the $x_{k}$ are numbered taking into account their multiplicity, then we have the identity 


$$
\begin{aligned}
f\left(x_{0}\right)=\sum_{k=1}^{r} f^{(2 k-1)}(a) \Psi_{2 k-1}^{*}(a) & -\sum_{k=1}^{r} f^{(2 k-1)}(-a) \Psi_{2 k-1}^{*}(-a) \\
& -\int_{-a}^{a} f^{(2 r)}(x) \Psi_{2 r-1}^{*}(x) d x
\end{aligned}
$$

where for short $\Psi_{2 k-1}^{*}( \pm a)$ and $\Psi_{2 r-1}^{*}(x)$ stand for $\Psi_{2 k-1}^{*}(\cdot, \ldots, \cdot, \pm a)$ and $\Psi_{2 r-1}^{*}(\cdot, \ldots, \cdot, x)$.

Proof. Introducing the function $h$ defined by

$$
h(t, x)=\frac{(4 a)^{2 l-1}}{(2 l) !}\left(B_{2 l}\left(\frac{1}{2}+\frac{x}{4 a}+\frac{1}{2 \pi} \operatorname{Arcsin} t\right)+B_{2 l}\left(\left\{\frac{x}{4 a}-\frac{1}{2 \pi} \operatorname{Arcsin} t\right\}\right)\right)
$$

we have

$$
\Psi_{2 l-1}\left(x_{-m}, \ldots, x_{n}, \pm a\right)=\frac{1}{\alpha_{0}} \sum_{k=-m}^{n} \alpha_{k} h\left(\sin \left(\pi \frac{x_{k}}{2 a}\right), \pm a\right)
$$

for pairwise distinct $\left.x_{-m}, \ldots, x_{n} \in\right]-a, a[$ and assertion a) holds since the functions $h(\cdot, \pm a)$ belong to $\left.C^{\infty}\right]-1,1[$.

By definition the function $h$ belongs to $C^{2 l-2}(]-1,1[\times[-a, a])$ and the assertion b) is a consequence of the representation formula (2.5) since we have $2 l-2 \geqslant$ $m+n$. For pairwise distinct $\left.x_{-m}, \ldots, x_{n} \in\right]-a, a[$ the left-hand side of identity (2.1) reads

$$
\frac{1}{\alpha_{0}} \sum_{k=-m}^{n} \alpha_{k} f\left(\frac{2 a}{\pi} \operatorname{Arcsin}\left(\sin \left(\pi \frac{x_{k}}{2 a}\right)\right)\right)
$$

and thanks to Lemma 2.2 this expression, and hence the identity (2.1), extend to $\left.x_{-m}, \ldots, x_{n} \in\right]-a, a[$. One completes the proof of c) by observing, thanks to Lemma 2.2. that the left-hand side reduces to $f\left(x_{0}\right)$ when the $x_{k}$ are zeros of multiplicity $r_{k}$ of $f$.

The results stated in Lemma 2.4 play a central role in the proof of properties of functions $\Psi_{2 l-1}^{*}(\cdot, \ldots, \cdot, \pm a)$.

Lemma 2.4. For all $m, k \in \mathbb{N}^{*}$ we have the inequality

$$
(-1)^{m+1} \frac{d^{k}}{d x^{k}} B_{2 m}\left(\frac{1}{2}+\frac{1}{\pi} \operatorname{Arcsin} \sqrt{x}\right)>0 \quad \text { for } x \in[0,1[.
$$

The proof of Lemma 2.4 requires two technical results given in Lemmas 2.5 and 2.6 .

Lemma 2.5. For all $k \in \mathbb{N}$ we have the Taylor expansion

$$
(\operatorname{Arcsin} x)^{2 k}=\sum_{l=0}^{\infty} \frac{(2 k) !}{(2 l) !} 2^{2 l-2 k} b_{k, l} x^{2 l} \quad \text { for } x \in[-1,1]
$$

where $b_{k, l}$ are integers defined recursively by 


$$
\begin{array}{cc}
b_{0,0}=1 \text { and } b_{k, 0}=b_{0, l}=0 & \text { for } k, l \geqslant 1 \\
b_{k+1, l+1}=b_{k, l}+l^{2} b_{k+1, l} & \text { for } k, l \geqslant 0 .
\end{array}
$$

Proof. We note first that the functions $f_{2 k}(x):=(\operatorname{Arcsin} x)^{2 k}$ satisfy

$\left(1-x^{2}\right) f_{2 k+2}^{\prime \prime}(x)-x f_{2 k+2}^{\prime}(x)-(2 k+2)(2 k+1) f_{2 k}(x)=0 \quad$ for $\left.x \in\right]-1,1[$.

From the definition of $f_{2 k}$ and the above equality it follows that numbers $c_{k, l}$ defined by $f_{2 k}(x)=\sum_{l=0}^{\infty} c_{k, l} x^{2 l}$ for $x \in[-1,1]$ are uniquely determined by the recurrence relations

$$
\begin{gathered}
c_{0,0}=1 \text { and } c_{k, 0}=c_{0, l}=0 \quad \text { for } k, l \geqslant 1 \\
(2 l+2)(2 l+1) c_{k+1, l+1}-4 l^{2} c_{k+1, l}-(2 k+2)(2 k+1) c_{k, l}=0 \quad \text { for } k, l \geqslant 0 .
\end{gathered}
$$

A simple check shows that $c_{k, l}=\frac{(2 k) !}{(2 l) !} 2^{2 l-2 k} b_{k, l}$.

Lemma 2.6. Let $b_{k, l}$ be the numbers defined in Lemma 2.5. Then

$$
\lim _{l \rightarrow \infty} \frac{b_{k, l}}{((l-1) !)^{2}}=\frac{\pi^{2 k-2}}{(2 k-1) !} \quad \text { for all } k \geqslant 1 \text {. }
$$

Proof. From the definition of numbers $b_{k, l}$ we infer that $b_{1, l}=((l-1) !)^{2}$ for $l \geqslant 1$. Thus relation (2.7) is trivially true for $k=1$. We then assume $k \geqslant 2$. As $b_{j, 1}=0$ for $j \geqslant 2$ the numbers $d_{j, l}$ defined for $j, l \geqslant 1$ by $d_{j, l}=\frac{b_{j, l}}{((l-1) !)^{2}}$ satisfy the recurrence relations

$$
\begin{aligned}
& d_{j, 1}=0 \text { and } d_{1, l}=1 \quad \text { for } j \geqslant 2 \text { and } l \geqslant 1, \\
& d_{j+1, l+1}=\frac{1}{l^{2}} d_{j, l}+d_{j+1, l} \quad \text { for } j, l \geqslant 1 .
\end{aligned}
$$

Using the fact that $d_{j-1, l}=0$ for $l=1, \ldots, j-2$ we get first for $j \geqslant 2$ the equality

$$
d_{j, n_{j}}=\sum_{n_{j-1}=j-1}^{n_{j}-1} \frac{1}{n_{j-1}^{2}} d_{j-1, n_{j-1}}
$$

which we iterate to obtain

$$
d_{k, l}=\sum_{n_{k-1}=k-1}^{l-1} \frac{1}{n_{k-1}^{2}} \sum_{n_{k-2}=k-2}^{n_{k-1}-1} \frac{1}{n_{k-2}^{2}} \cdots \sum_{n_{2}=2}^{n_{3}-1} \frac{1}{n_{2}^{2}} \sum_{n_{1}=1}^{n_{2}-1} \frac{1}{n_{1}^{2}} .
$$

This leads to

$$
\lim _{l \rightarrow \infty} d_{k, l}=\sum_{n_{k-1}>n_{k-2}>\cdots>n_{2}>n_{1}>0} \prod_{j=1}^{k-1} \frac{1}{n_{j}^{2}}
$$

and we recognize in the right-hand side the number $\zeta\left(\{2\}_{(k-1)}\right)$ whose value, given in [2], is equal to the right-hand side of (2.7). 
Proof of Lemma 2.4. It suffices to prove that the numbers $e_{m, l}$ defined by

$$
(-1)^{m+1} B_{2 m}\left(\frac{1}{2}+\frac{1}{\pi} \operatorname{Arcsin} x\right)=\sum_{l=0}^{\infty} e_{m, l} x^{2 l}
$$

satisfy $e_{m, l}>0$ for all $m, l \in \mathbb{N}^{*}$. Using Taylor's formula and the evenness of function $B_{2 m}\left(\frac{1}{2}+\frac{t}{\pi}\right)$, we have

$$
B_{2 m}\left(\frac{1}{2}+\frac{t}{\pi}\right)=\sum_{k=0}^{m} \frac{1}{(2 k) !} B_{2 m}^{(2 k)}\left(\frac{1}{2}\right)\left(\frac{t}{\pi}\right)^{2 k}=\sum_{k=0}^{m}\left(\begin{array}{c}
2 m \\
2 k
\end{array}\right) B_{2 m-2 k}\left(\frac{1}{2}\right)\left(\frac{t}{\pi}\right)^{2 k}
$$

and the Taylor expansion of $(\operatorname{Arcsin} x)^{2 k}$ given in Lemma 2.5 leads to

$$
\begin{aligned}
B_{2 m}\left(\frac{1}{2}+\frac{1}{\pi} \operatorname{Arcsin} x\right) & =\sum_{k=0}^{m}\left(\left(\begin{array}{c}
2 m \\
2 k
\end{array}\right) B_{2 m-2 k}\left(\frac{1}{2}\right) \pi^{-2 k} \sum_{l=0}^{\infty} \frac{(2 k) !}{(2 l) !} 2^{2 l-2 k} b_{k, l} x^{2 l}\right) \\
& =\frac{(2 m) !}{(2 \pi)^{2 m}} \sum_{k=0}^{m}\left(\frac{(2 \pi)^{2 m-2 k}}{(2 m-2 k) !} B_{2 m-2 k}\left(\frac{1}{2}\right) \sum_{l=0}^{\infty} \frac{2^{2 l}}{(2 l) !} b_{k, l} x^{2 l}\right)
\end{aligned}
$$

We then change the order of summation to get

$$
(-1)^{m+1} B_{2 m}\left(\frac{1}{2}+\frac{1}{\pi} \operatorname{Arcsin} x\right)=\frac{(2 m) !}{(2 \pi)^{2 m}} \sum_{l=0}^{\infty} \frac{2^{2 l}}{(2 l) !} f_{m, l} x^{2 l}
$$

where

$$
f_{m, l}=(-1)^{m+1} \sum_{k=0}^{m} \frac{(2 \pi)^{2 m-2 k}}{(2 m-2 k) !} B_{2 m-2 k}\left(\frac{1}{2}\right) b_{k, l} .
$$

We prove by recurrence over $m$ that $f_{m, l}>0$ for $m, l \geqslant 1$. To this end we set $g_{m, l}=\frac{f_{m, l}}{((l-1) !)^{2}}$ for $m, l \geqslant 1$ and since $b_{0, l}=0$ for $l \geqslant 1$ we have

$$
\begin{aligned}
g_{m+1, l+1} & =\frac{(-1)^{m+2}}{(l !)^{2}} \sum_{k=1}^{m+1} \frac{(2 \pi)^{2 m+2-2 k}}{(2 m+2-2 k) !} B_{2 m+2-2 k}\left(\frac{1}{2}\right) b_{k, l+1} \\
& =\frac{(-1)^{m+2}}{(l !)^{2}} \sum_{k=1}^{m+1} \frac{(2 \pi)^{2 m+2-2 k}}{(2 m+2-2 k) !} B_{2 m+2-2 k}\left(\frac{1}{2}\right)\left(b_{k-1, l}+l^{2} b_{k, l}\right) \\
& =\frac{(-1)^{m+2}}{(l !)^{2}} \sum_{k=1}^{m+1} \frac{(2 \pi)^{2 m+2-2 k}}{(2 m+2-2 k) !} B_{2 m+2-2 k}\left(\frac{1}{2}\right) b_{k-1, l}+g_{m+1, l} \\
& =-\frac{(-1)^{m+1}}{(l !)^{2}} \sum_{k=0}^{m} \frac{(2 \pi)^{2 m-2 k}}{(2 m-2 k) !} B_{2 m-2 k}\left(\frac{1}{2}\right) b_{k, l}+g_{m+1, l} \\
& =-\frac{1}{l^{2}} g_{m, l}+g_{m+1, l}
\end{aligned}
$$

and this implies that

$$
g_{m+1, l+1}+\frac{1}{l^{2}} g_{m, l}=g_{m+1, l} \text { for } l \geqslant 1 .
$$


We have $g_{1, l}=f_{1, l}=1$ for all $l \geqslant 1$. Let us suppose that $g_{m, l}>0$ for all $l \geqslant 1$. Then $g_{m+1, l+1}<g_{m+1, l}$ and it follows that $g_{m+1, l}>\lim _{l \rightarrow \infty} g_{m+1, l}$. Thanks to Lemma 2.6 we have

$$
\begin{aligned}
\lim _{l \rightarrow \infty} g_{m+1, l} & =(-1)^{m+2} \sum_{k=1}^{m+1} \frac{(2 \pi)^{2 m+2-2 k}}{(2 m+2-2 k) !} B_{2 m+2-2 k}\left(\frac{1}{2}\right) \frac{\pi^{2 k-2}}{(2 k-1) !} \\
& =(-1)^{m+2} \pi^{2 m} \sum_{k=1}^{m+1} \frac{2^{2 m+2-2 k}}{(2 m+2-2 k) !(2 k-1) !} B_{2 m+2-2 k}\left(\frac{1}{2}\right)
\end{aligned}
$$

and using $B_{j}\left(\frac{1}{2}\right)=0$ for all odd $j$ and the formula

$$
B_{n}(x+y)=\sum_{j=0}^{n}\left(\begin{array}{l}
n \\
j
\end{array}\right) B_{j}(x) y^{n-j}
$$

we check that the sum which appears in (2.10) is equal to

$$
\begin{aligned}
\sum_{j=0}^{2 m+1} \frac{2^{j}}{j !(2 m+1-j) !} B_{j}\left(\frac{1}{2}\right) & =\frac{2^{2 m+1}}{(2 m+1) !} \sum_{j=0}^{2 m+1}\left(\begin{array}{c}
2 m+1 \\
j
\end{array}\right) B_{j}\left(\frac{1}{2}\right)\left(\frac{1}{2}\right)^{2 m+1-j} \\
& =\frac{2^{2 m+1}}{(2 m+1) !} B_{2 m+1}(1)=0 .
\end{aligned}
$$

Hence $g_{m, l}>0$ for $m, l \geqslant 1$ and this implies, thanks to (2.9), that the numbers $e_{m, l}$ defined by (2.8) are positive for $m, l \geqslant 1$.

We are now in position to prove main properties of functions $\Psi_{2 l-1}^{*}(\cdot, \ldots, \cdot, \pm a)$.

Lemma 2.7. Let $\Psi_{2 l-1}^{*}(\cdot, \ldots, \cdot, \pm a)$ be the functions defined in Lemma 2.3 . Then

a) $(-1)^{n+l+1} \Psi_{2 l-1}^{*}\left(x_{-m}, \ldots, x_{n}, a\right)>0$ for $\left.x_{-m}, \ldots, x_{n} \in\right]-a, a[$.

b) $(-1)^{m+l+1} \Psi_{2 l-1}^{*}\left(x_{-m}, \ldots, x_{n},-a\right)>0$ for $\left.x_{-m}, \ldots, x_{n} \in\right]-a, a[$.

Proof. For pairwise distinct $\left.x_{-m}, \ldots, x_{n} \in\right]-a, a[$ we have

$$
\Psi_{2 l-1}^{*}\left(x_{-m}, \ldots, x_{n}, \pm a\right)=2 \frac{(4 a)^{2 l-1}}{(2 l) !} \sum_{k=-m}^{n} \mu_{k} B_{2 l}\left(\frac{1}{2}+\frac{ \pm a+x_{k}}{4 a}\right)
$$

since the function $B_{2 m}\left(\frac{1}{2}+t\right)$ is even and then

$$
\begin{aligned}
& (-1)^{n+l+1} \Psi_{2 l-1}^{*}\left(x_{-m}, \ldots, x_{n}, \pm a\right) \\
& =2 \frac{(4 a)^{2 l-1}}{(2 l) !}\left(\frac{(-1)^{n}}{\alpha_{0}}\right) \sum_{k=-m}^{n} \alpha_{k}(-1)^{l+1} B_{2 l}\left(\frac{1}{2}+\frac{ \pm a+x_{k}}{4 a}\right) .
\end{aligned}
$$


The first two terms of the right-hand side are positive and the third term reads $\sum_{k=-m}^{n} \alpha_{k} h_{ \pm}\left(\sin \left(\pi \frac{x_{k}}{2 a}\right)\right)$ where

$$
h_{ \pm}(t)=(-1)^{l+1} B_{2 l}\left(\frac{3}{4} \pm \frac{1}{2 \pi} \operatorname{Arcsin} t\right) \quad \text { for } t \in[-1,1] .
$$

The identities

$$
\frac{3}{4} \pm \frac{1}{2 \pi} \operatorname{Arcsin} t=\frac{1}{2}+\frac{1}{\pi} \operatorname{Arcsin} \sqrt{\frac{1 \pm t}{2}} \text { for } t \in[-1,1]
$$

together with Lemma 2.4 show that $h_{+}^{(m+n)}$ and $(-1)^{m+n} h_{-}^{(m+n)}$ are positive on ] $-1,1$ [ and the conclusion holds by Lemma 2.2 .

The last point is to bound the integral which appears in the right-hand side of the identity (2.6). This is the content of Lemma 2.9. whose proof needs the following result.

LEMma 2.8. Let $b_{r, s}$ the numbers defined for integers $r \geqslant 4$ and $s \geqslant 0$ by

$$
b_{r, s}=\left(\frac{r}{r+s}\right)^{2 r \log r-1}\left(\begin{array}{c}
2 r+s-1 \\
s
\end{array}\right) .
$$

Then $\sum_{s=0}^{\infty} b_{r, s}^{2}=1+o(1)$ as $r \rightarrow \infty$.

Proof. We have $b_{r, s}=O_{r}\left(s^{-2 r \log r+2 r}\right)=O_{r}\left(s^{-1}\right)$ and hence $\sum_{s=0}^{\infty} b_{4, s}^{2}$ is convergent. We now prove that $b_{r, s} \leqslant b_{4, s}$ for $r \geqslant 4$. We have $\log b_{r, s}=g(r, s)$ where the function $g$ is defined for $(x, y) \in[4, \infty[\times[0, \infty[$ by

$$
g(x, y)=(2 x \log x-1) \log \left(\frac{x}{x+y}\right)+\log \Gamma(2 x+y)-\log \Gamma(y+1)-\log \Gamma(2 x) .
$$

Straightforward computations lead to

$\frac{\partial g}{\partial x}(x, y)=(2 \log x+2) \log \left(\frac{x}{x+y}\right)+(2 x \log x-1) \frac{y}{x(x+y)}+2 \Psi(2 x+y)-2 \Psi(2 x)$

and

$$
\frac{\partial^{2} g}{\partial y \partial x}(x, y)=-\frac{1+2 x+2 y+2 y \log x}{(x+y)^{2}}+2 \Psi^{\prime}(2 x+y)
$$

where $\Psi$ is the derivative of $\log \Gamma$. We have $\frac{\partial g}{\partial x}(x, 0)=0$ and moreover since $\Psi^{\prime}(z)=\sum_{k=0}^{\infty} \frac{1}{(z+k)^{2}}$, we get $\Psi^{\prime}(z) \leqslant \frac{1}{z}+\frac{1}{z^{2}}$ for $z>0$ and therefore

$$
\begin{aligned}
\frac{\partial^{2} g}{\partial y \partial x}(x, y) & \leqslant-\frac{1+2 x+2 y+2 y \log x}{(x+y)^{2}}+\frac{2}{2 x+y}+\frac{2}{(2 x+y)^{2}} \\
& =-\left(\frac{4 x^{3}+2(1+3 y) x^{2}+(2 x-1) y^{2}}{(x+y)^{2}(2 x+y)^{2}}+\frac{2 y \log x}{(x+y)^{2}}\right) \leqslant 0 .
\end{aligned}
$$

Hence $\frac{\partial g}{\partial x}(x, y) \leqslant \frac{\partial g}{\partial x}(x, 0)=0$ and this implies that $g(x, y) \leqslant g(4, y)$ and hence $b_{r, s} \leqslant b_{4, s}$ for $r \geqslant 4$. 
Let $\epsilon>0$ and $s_{0}$ be such that $\sum_{s=s_{0}}^{\infty} b_{4, s}^{2} \leqslant \frac{\epsilon}{2}$. Since $b_{r, s} \rightarrow 0$ as $r \rightarrow \infty$ for $s \geqslant 1$, there exists $r_{0}$ such that $\sum_{s=1}^{s_{0}-1} b_{r, s}^{2} \leqslant \frac{\epsilon}{2}$ for $r \geqslant r_{0}$. Hence

$$
1 \leqslant \sum_{s=0}^{\infty} b_{r, s}^{2} \leqslant b_{r, 0}^{2}+\sum_{s=1}^{s_{0}-1} b_{r, s}^{2}+\sum_{s=s_{0}}^{\infty} b_{4, s}^{2} \leqslant 1+\epsilon \quad \text { for } r \geqslant r_{0} .
$$

The proof is complete.

LEMма 2.9. For positive integers $m, n$ and $l$ which satisfy $m+n \geqslant 4$ and $l \geqslant(m+n) \log (m+n)$ and for $\left.x_{-m}, \ldots, x_{n} \in\right]-a, a\left[\right.$, let $\Psi_{2 l-1}^{*}$ be the function defined in Lemma 2.3. Then

$$
\left\|\Psi_{2 l-1}^{*}\right\|_{2}=\frac{2^{m+n-1}}{\left|\alpha_{0}\right| \sqrt{a}}\left(\frac{2 a}{(m+n) \pi}\right)^{2 l}(1+o(1)) \quad \text { as } m+n \rightarrow \infty
$$

where

$$
\left\|\Psi_{2 l-1}^{*}\right\|_{2}^{2}=\int_{-a}^{a}\left(\Psi_{2 l-1}^{*}\left(x_{-m}, \ldots, x_{n}, x\right)\right)^{2} d x .
$$

Proof. The use of the Fourier series expansion

$$
B_{2 l}(x)=(-1)^{l+1} 2((2 l) !) \sum_{j=1}^{\infty} \frac{1}{(2 j \pi)^{2 l}} \cos (2 j \pi x) \quad \text { for } x \in[0,1]
$$

and the identity $\cos \alpha+\cos \beta=2 \cos \left(\frac{\alpha+\beta}{2}\right) \cos \left(\frac{\alpha-\beta}{2}\right)$ lead, for pairwise distinct $\left.x_{-m}, \ldots, x_{n} \in\right]-a, a[$, to the expression

$$
\begin{aligned}
& \Psi_{2 l-1}^{*}\left(x_{-m}, \ldots, x_{n}, x\right) \\
= & (-1)^{l+1} 2 \frac{(2 a)^{2 l-1}}{\alpha_{0} \pi^{2 l}} \sum_{j=1}^{\infty} \frac{1}{j^{2 l}}\left(\sum_{k=-m}^{n} \alpha_{k} \cos \left(j \pi\left(\frac{1}{2}+\frac{x_{k}}{2 a}\right)\right)\right) \cos \left(j \pi\left(\frac{1}{2}+\frac{x}{2 a}\right)\right) .
\end{aligned}
$$

Using the identity $\cos \left(j \pi\left(\frac{1}{2}+y\right)\right)=(-1)^{j} T_{j}(\sin (\pi y))$ and introducing the numbers $a_{j, k}=(-1)^{j} T_{j}\left(\sin \left(\pi \frac{x_{k}}{2 a}\right)\right)$ we have $\sum_{k=-m}^{n} \alpha_{k} a_{j, k}=0$ for $j=1, \ldots, m+n-1$, this is crucial, and, therefore

$$
\begin{aligned}
& \Psi_{2 l-1}^{*}\left(x_{-m}, \ldots, x_{n}, x\right) \\
& \quad=(-1)^{l+1} 2 \frac{(2 a)^{2 l-1}}{\alpha_{0} \pi^{2 l}} \sum_{j=m+n}^{\infty} \frac{1}{j^{2 l}}\left(\sum_{k=-m}^{n} \alpha_{k} a_{j, k}\right) \cos \left(j \pi\left(\frac{1}{2}+\frac{x}{2 a}\right)\right) .
\end{aligned}
$$

Using Lemma 2.2, squaring (2.11) and integrating on the interval $[-a, a]$, we get

$$
\left\|\Psi_{2 l-1}^{*}\right\|_{2}^{2}=\left(2 \frac{(2 a)^{2 l-1}}{\alpha_{0} \pi^{2 l}}\right)^{2} \sum_{j=m+n}^{\infty} \frac{1}{j^{4 l}}\left(\frac{T_{j}^{(m+n)}\left(\tau_{j}\right)}{(m+n) !}\right)^{2} a
$$

for some $\left.\tau_{j} \in\right]-1,1[$. It is well known [10] that

$$
\max _{-1 \leqslant x \leqslant 1}\left|T_{j}^{(m+n)}(x)\right|=T_{j}^{(m+n)}(1)=2^{m+n-1}(m+n-1) ! j\left(\begin{array}{c}
m+n+j-1 \\
j-m-n
\end{array}\right)
$$


for $j=m+n, m+n+1, \ldots$ and then

$$
\left\|\Psi_{2 l-1}^{*}\right\|_{2}^{2} \leqslant\left(\frac{2^{m+n-1}}{\alpha_{0} \sqrt{a}}\left(\frac{2 a}{(m+n) \pi}\right)^{2 l}\right)^{2} \sum_{j=m+n}^{\infty}\left(\frac{m+n}{j}\right)^{4 l-2}\left(\begin{array}{c}
m+n+j-1 \\
j-m-n
\end{array}\right)^{2} .
$$

We set $j=m+n+s$ and since $l \geqslant(m+n) \log (m+n)$ we have

$$
\begin{aligned}
\left\|\Psi_{2 l-1}^{*}\right\|_{2}^{2} & \leqslant\left(\frac{2^{m+n-1}}{\alpha_{0} \sqrt{a}}\left(\frac{2 a}{(m+n) \pi}\right)^{2 l}\right)^{2} \sum_{s=0}^{\infty}\left(\left(\frac{m+n}{m+n+s}\right)^{2 l-1}\left(\begin{array}{c}
2(m+n)+s-1 \\
s
\end{array}\right)\right)^{2} \\
& \leqslant\left(\frac{2^{m+n-1}}{\alpha_{0} \sqrt{a}}\left(\frac{2 a}{(m+n) \pi}\right)^{2 l}\right)^{2} \sum_{s=0}^{\infty} b_{m+n, s}^{2} \\
& =\left(\frac{2^{m+n-1}}{\alpha_{0} \sqrt{a}}\left(\frac{2 a}{(m+n) \pi}\right)^{2 l}\right)^{2}(1+o(1))
\end{aligned}
$$

as $m+n \rightarrow \infty$, thanks to Lemma 2.8. To complete the proof we compute a lower bound for $\left\|\Psi_{2 l-1}^{*}\right\|_{2}^{2}$ using the first term in the series which appears in the right-hand side of (2.12).

\section{Proof of Theorem}

In this section we assume that the Riemann hypothesis is true. Our result is a consequence of identity (2.6) which for $Z(T)$ gives

$$
\begin{aligned}
Z(T)=\sum_{k=1}^{K} Z^{(2 k-1)}(T+a) \Psi_{2 k-1}^{*}(a) & -\sum_{k=1}^{K} Z^{(2 k-1)}(T-a) \Psi_{2 k-1}^{*}(-a) \\
& -\int_{-a}^{a} Z^{(2 K)}(T+x) \Psi_{2 K-1}^{*}(x) d x
\end{aligned}
$$

where $2 K \geqslant m+n+2$ and for short $\Psi_{2 k-1}^{*}( \pm a)$ and $\Psi_{2 m-1}^{*}(x)$ stand respectively for $\Psi_{2 k-1}^{*}\left(x_{-m}, \ldots, x_{n}, \pm a\right)$ and $\Psi_{2 m-1}^{*}\left(x_{-m}, \ldots, x_{n}, x\right)$ and $x_{k}=\gamma_{k}-T$. The main step in the proof is to bound the integral which appears in the right-hand side of (3.1). In our proof we use the bound

$$
\left|\int_{T}^{T+h} S(u) d u\right| \leqslant \frac{\pi}{16} \frac{\log T}{(\log \log T)^{2}}+O\left(\frac{\log T \log \log \log T}{(\log \log T)^{3}}\right)
$$

for $0<h \leqslant \sqrt{T}$, due to Carneiro et al. [3].

Lemma 3.1. Let $T$ be sufficiently large, $4(\log \log T)^{-1} \leqslant a \leqslant \sqrt{T}, m$ and $n$ be the integers defined in the introduction and let $\alpha_{0}$ be the coefficient defined in Lemma 2.3 where $x_{0}=0$ and $x_{k}=\gamma_{k}-T$. Then

$$
-\log \left|\alpha_{0}\right| \leqslant-\frac{2 a}{\pi} \theta^{\prime}(T) \log 2+\frac{1}{\sqrt{2}} \frac{\log T}{\log \log T}\left(1+O\left(\frac{\log \log \log T}{\log \log T}\right)\right) .
$$


Further, if $S(T+a)-S(T-a) \geqslant 1$, then

$$
m+n=\frac{2 a}{\pi} \theta^{\prime}(T)+\Delta S\left(1+O\left(\frac{a^{3}}{T^{2}}\right)\right)
$$

where $\Delta S=S(T+a)-S(T-a)$.

Proof. By definition

$$
\left|\frac{1}{\alpha_{0}}\right|=\prod_{\substack{-m \leqslant j \leqslant n \\ j \neq 0}}\left|\sin \left(\pi \frac{x_{j}}{2 a}\right)\right|
$$

and using (1.1) and Stieltjes integral we have

$$
-\log \left|\alpha_{0}\right|=\int_{T-a}^{T+a} \log \left|\sin \left(\pi \frac{t-T}{2 a}\right)\right| d\left(\frac{1}{\pi} \theta(t)+1+S(t)\right)
$$

and an integration by parts leads to

$$
\begin{aligned}
-\log \left|\alpha_{0}\right|= & \frac{1}{\pi} \int_{T-a}^{T+a} \theta^{\prime}(t) \log \left|\sin \left(\pi \frac{t-T}{2 a}\right)\right| d t \\
& -\frac{\pi}{2 a} \int_{T-a}^{T+a} \cot \left(\pi \frac{t-T}{2 a}\right)(S(t)-S(T)) d t
\end{aligned}
$$

Now for $t \in[T-a, T+a]$ we have

$$
\theta^{\prime}(t)=\theta^{\prime}(T)+\theta^{\prime \prime}(T)(t-T)+\frac{1}{2} \theta^{\prime \prime \prime}(\tau)(t-T)^{2} \quad \text { for some } \tau \in[T-a, T+a]
$$

and using $\theta^{\prime \prime \prime}(t)=O\left(1 / t^{2}\right)$ together with

$$
\int_{T-a}^{T+a} \log \left|\sin \left(\pi \frac{t-T}{2 a}\right)\right| d t=\frac{4 a}{\pi} \int_{0}^{\pi / 2} \log \sin \tau d \tau=-2 a \log 2
$$

we get

$$
\begin{aligned}
-\log \left|\alpha_{0}\right|= & -\frac{2 a}{\pi} \theta^{\prime}(T) \log 2 \\
& -\frac{\pi}{2 a} \int_{T-a}^{T+a} \cot \left(\pi \frac{t-T}{2 a}\right)(S(t)-S(T)) d t+O\left(\frac{a^{3}}{T^{2}}\right) .
\end{aligned}
$$

Further, for $\eta \in] 0, a]$ we have

$$
\begin{aligned}
\int_{T-a}^{T+a} \cot & \left(\pi \frac{t-T}{2 a}\right)(S(t)-S(T)) d t \\
= & \int_{T-a}^{T-\eta} \cot \left(\pi \frac{t-T}{2 a}\right) S(t) d t+\int_{T-\eta}^{T} \cot \left(\pi \frac{t-T}{2 a}\right)(S(t)-S(T)) d t \\
& +\int_{T}^{T+\eta} \cot \left(\pi \frac{t-T}{2 a}\right)(S(t)-S(T)) d t+\int_{T+\eta}^{T+a} \cot \left(\pi \frac{t-T}{2 a}\right) S(t) d t
\end{aligned}
$$


and we bound from below the third term using

$$
S(t)-S(T) \geqslant-\frac{1}{\pi}(\theta(t)-\theta(T)) \geqslant-\frac{1}{\pi} \theta^{\prime}(T+a)(t-T) \geqslant-\frac{1}{2 \pi} \log T(t-T)
$$

which, together with the inequality $\cot x<\frac{1}{x}$ for $\left.\left.x \in\right] 0, \frac{\pi}{2}\right]$, implies

$$
\int_{T}^{T+\eta} \cot \left(\pi \frac{t-T}{2 a}\right)(S(t)-S(T)) d t \geqslant-\frac{\eta a}{\pi^{2}} \log T .
$$

Applied to the fourth term, the second mean-value theorem gives

$$
\begin{aligned}
\int_{T+\eta}^{T+a} \cot \left(\pi \frac{t-T}{2 a}\right) S(t) d t & =\cot \left(\pi \frac{\eta}{2 a}\right) \int_{T+\eta}^{\tau} S(t) d t \\
& \geqslant-\frac{2 a}{\pi \eta}\left(\frac{\pi}{16} \frac{\log T}{(\log \log T)^{2}}+O\left(\frac{\log T \log \log \log T}{(\log \log T)^{3}}\right)\right)
\end{aligned}
$$

where $\tau \in[T+\eta, T+a]$ and (3.2) has been used. Proceeding in the same way to bound from below the first and second term we finally get

$$
\begin{aligned}
& -\frac{\pi}{2 a} \int_{T-a}^{T+a} \cot \left(\pi \frac{t-T}{2 a}\right)(S(t)-S(T)) d t \\
& \quad \leqslant \frac{\eta}{\pi} \log T+\frac{1}{\eta}\left(\frac{\pi}{8} \frac{\log T}{(\log \log T)^{2}}+O\left(\frac{\log T \log \log \log T}{(\log \log T)^{3}}\right)\right) .
\end{aligned}
$$

We choose $\eta=\pi(2 \sqrt{2} \log \log T)^{-1}$ and we complete the proof of (3.3) using (3.5). Since $m+n=N(T+a)-N(T-a)$, the Taylor formula leads to

$$
m+n=\frac{1}{\pi}(\theta(T+a)-\theta(T-a))+\Delta S=\frac{2 a}{\pi} \theta^{\prime}(T)+\Delta S\left(1+O\left(\frac{a^{3}}{T^{2}}\right)\right) .
$$

which proves (3.4).

Proof of TheOrem [1.1. Either $K \leqslant K^{*} \log K^{*}$ and there is nothing to prove or $K>K^{*} \log K^{*}$ and this implies that $K \geqslant(m+n) \log (m+n)$. Assuming $T$ large enough we have also $m+n \geqslant 4$. By definition of $K$ and thanks to Lemma 2.7 the first sum in the right-hand side of identity (3.1) is nonpositive and the second one is nonnegative. Using Cauchy-Schwarz inequality, (1.4) and Lemma 2.9, we get

$$
Z(T) \leqslant\left\|Z^{(2 K)}\right\|_{2}\left\|\Psi_{2 K-1}^{*}\right\|_{2} \leqslant C_{2 K} Z(T)
$$

where

$$
\begin{aligned}
C_{2 K} & =c_{2 K} 2^{m+n-\frac{1}{2}}\left|\alpha_{0}\right|^{-1}\left(\frac{2 a \theta^{\prime}(T)}{(m+n) \pi}\right)^{2 K}(1+o(1)) \\
& \leqslant c_{2 K} 2^{m+n}\left|\alpha_{0}\right|^{-1}\left(1+\pi \frac{\Delta S}{2 a \theta^{\prime}(T)}\left(1+O\left(\frac{a^{3}}{T^{2}}\right)\right)\right)^{-2 K}
\end{aligned}
$$


for $T$ sufficiently large. Since $C_{2 K} \geqslant 1$ we have $\log C_{2 K} \geqslant 0$ and this implies that

$$
\log c_{2 K}+(m+n) \log 2-\log \left|\alpha_{0}\right|-2 K \log \left(1+\pi \frac{\Delta S}{2 a \theta^{\prime}(T)}\left(1+O\left(\frac{a^{3}}{T^{2}}\right)\right)\right) \geqslant 0 .
$$

Finally, as $\Delta S=O\left(\log T(\log \log T)^{-1}\right)$ and thanks to Lemma 3.1, we get

$$
\begin{aligned}
& 2 K \pi \frac{\Delta S}{2 a \theta^{\prime}(T)}(1\left.+O\left(\frac{1}{\log \log T}\right)\right) \\
& \leqslant\left(\log c_{2 K}+\Delta S \log 2+\frac{1}{\sqrt{2}} \frac{\log T}{\log \log T}\right)\left(1+O\left(\frac{\log \log \log T}{\log \log T}\right)\right)
\end{aligned}
$$

and the proof is complete.

Acknowledgements. I am grateful to Professor Jean Descloux for his encouragements, to my colleagues Jean-François Hêche, Fred Lang, Eric Thiémard and Jacques Zuber for their help and to my wife, Ariane, a very creative artist.

\section{References}

1. Ph. Blanc, Lower bound for the maximum of some derivative of Hardy's function, Report 11.2013 - Mathicse - EPFL

2. J. M. Borwein, D. M. Bradley, D. J. Broadhurst, Evaluations of k-fold Euler/Zagier sums : A compendium of results for arbitrary $k$, J. Combin. 4(2) 1997, 31-49.

3. E. Carneiro, V. Chandee, M. B. Milinovich, Bounding $S(t)$ and $S 1(t)$ on the Riemann hypothesis, Math. Ann. 356(3) (2013), 939-968.

4. D. A. Goldston, S. M. Gonek, A note on $S(t)$ and the zeros of the Riemann zeta-function, Bull. Lond. Math. Soc. 39 (2007), 482-486.

5. A. Ivić, On some reasons for doubting the Riemann hypothesis, in: P. Borwein, S. Choi, B. Rooney, A. Weirathmueller (Eds), The Riemann Hypothesis: a Resource for the Afficionado and Virtuoso Alike, Société mathématique du Canada, 2008, 130-160.

6. The Theory of Hardy's Z-function, Camb. Tracts Math. 196, Cambridge University Press, 2013

7. T. Kotnik, Computational estimation of the order of $\zeta\left(\frac{1}{2}+i t\right)$, Math. Comp. 73 (2004), 949-956.

8. A. A. Lavrik, Uniform approximations and zeros in short intervals of the derivatives of the Hardy's Z-function, (in Russian) Anal. Math. 17(4) (1991), 257-279.

9. O. T. Pop, D. Barbosu, Two dimensional divided differences with multiple knots, An. St. Univ. Ovidius Constanta, Ser. Mat. 17(2) (2009), 181-190.

10. T. J. Rivlin, Chebyshev Polynomials, Wiley, New York, 1990.

Département des technologies industrielles

(Received 1912 2013)

Haute École d'Ingénierie et de Gestion

CH-1400 Yverdon-les-Bains

Switzerland

philippe.blanc@heig-vd.ch 